\title{
Screening of latent tuberculosis infection among healthcare students and medical doctors using the tuberculin skin test
}

\author{
REDA GOWEDA ${ }^{1,2, A-F}$ \\ ORCID ID: 0000-0001-5514-1407 \\ ${ }^{1}$ Department of Family Medicine, Faculty of Medicine, Suez Canal University, Ismailia, Egypt \\ ${ }^{2}$ Department of Community Medicine, Faculty of Medicine, Umm Al-Qura University, Makkah, \\ Kingdom of Saudi Arabia
}

A - Study Design, B - Data Collection, C - Statistical Analysis, D - Data Interpretation, E - Manuscript Preparation, F - Literature Search, G - Funds Collection

Summary Background. Latent tuberculosis infection (LTBI) is defined as the absence of symptoms of tuberculosis in individuals who have an immune response stimulated by Mycobacterium tuberculosis antigens.

Objectives. To estimate the prevalence of LTBI among healthcare students and medical doctors using the tuberculin skin test and to study the associated risk factors.

Material and methods. This cross-sectional study was carried out to review the tuberculin skin test (TST) records of newly hired medical doctors, medical interns and medical students. A review of pre-employment records was done, including results of TST and sociodemographic information, as well as potential factors influencing TST reaction.

Results. Out of 186 enrolled personnel, 43 (23.1\%) were positive for TST. A positive TST was significantly associated with high BM (OR $=1.07,95 \% \mathrm{Cl}: 1.003-1.142, p=0.039$ ), while it was not significantly associated with male gender (OR $=1.195,95 \% \mathrm{Cl}: 0.530$ $-2.694, p=0.668)$, age $(\mathrm{OR}=0.973,95 \% \mathrm{Cl}: 0.915-1.034, p=0.371)$, smoking ( $\mathrm{OR}=0.730,95 \% \mathrm{Cl}: 0.259-2.060, p=0.552)$, $\mathrm{BCG}$ vaccination (OR $=0.785,95 \% \mathrm{Cl}: 0.249-2.481, p=0.680)$ or chronic disease $(\mathrm{OR}=4.828,95 \% \mathrm{Cl}: 0.910-25.608, p=0.064)$.

Conclusions. Our study population was at a significantly higher risk of LTBI, thus infection control precautions are strongly recommended. Furthermore, more studies are needed to assess the adherence and practices of healthcare workers, especially those who are exposed to tuberculosis patients.

Key words: tuberculosis, infection, mass screening.

Goweda R. Screening of latent tuberculosis infection among healthcare students and medical doctors using the tuberculin skin test. Fam Med Prim Care Rev 2021; 23(2): 163-168, doi: https://doi.org/10.5114/fmpcr.2021.105908.

\section{Background}

Latent tuberculosis infection (LTBI) is caused by Mycobacterium tuberculosis, leading to stimulation of the immune system and a positive tuberculin skin test reaction [1]. A patient with LTBI is asymptomatic and non-infectious to others. However, they have a $5-10 \%$ life-time risk of developing active tuberculosis (TB) [2].

In 2016, the World Health Organization [WHO] reported that 1.7 billion people had LTBI worldwide and estimated the prevalence of LTBI to be $23 \%$ [3]. International research and research work in Saudi Arabia have indicated an increased risk of tuberculosis as a re-emerging disease, with healthcare workers (HCWs) at particular high risk of exposure to tuberculosis infection. Despite being an old disease, TB continues to be a major public health concern, especially in low- and middle-income settings, such as the Middle East and other developing countries and regions [4].

LTBI screening in high risk individuals is strongly recommended in order to prevent the spread of TB. Accordingly, HCWs have a higher risk of contracting TB than the general population through occupational exposure and should be screened for TB [5]. Routine screening of the general population and not-at-risk groups is not recommended due to the waste of resources, as well as false-positive reactions leading to unnecessary anxiety [6].

TST has traditionally been used as a screening tool for LTBI due to low direct costs and ease of use [7]. Local Saudi guide- lines on the management of latent tuberculosis do not recommend using the Interferon Gamma Release Assays (IGRAs) for the diagnosis of active tuberculosis. Interpretation of both tests must be based on the patient's immune status, history of exposure to TB and bacillus Calmette-Guérin [BCG], as well as other risk factors [8].

The results of TST should be interpreted cautiously, and the result is individualized according to risk factors. There are three cut off points of TST reaction [ $5 \mathrm{~mm}$ induration, $10 \mathrm{~mm}$ induration or $15 \mathrm{~mm}$ induration]. A $5 \mathrm{~mm}$ or more induration is considered positive in the following: HIV-positive patients, personnel with recent contact with an active TB patient, individuals with nodular or fibrotic changes on chest X-ray, patients with organ transplants and other immuno-incompetent patients. An induration of $\geq 10 \mathrm{~mm}$ is considered positive in healthcare workers, recent arrivals (less than five years) from high-prevalence regions, injection drug users, high-risk congregational settings (e.g. prisons, hospitals, homeless, etc.), bacteriology laboratory personnel, along with some medical conditions, such as diabetes mellitus, prolonged corticosteroid therapy, leukemia, renal failure, etc. Finally $\geq 15 \mathrm{~mm}$ is considered positive in individuals with no known risk factors for TB $[9,10]$.

\section{Objectives}

To estimate the prevalence of LTBI among healthcare students and medical doctors using the tuberculin skin test and to study the associated risk factors. 


\section{Material and methods}

\section{Study design and setting}

This cross-sectional study was carried out to review the TST records of the newly hired medical doctors in Makkah city, as well as medical interns and medical students of Umm Al-Qura University, Saudi Arabia, between February 2020 and May 2020.

According to Saudi guidelines, newly hired physicians, medical intern and students receiving practical training in hospitals should be tested for LTBI. Additionally, a pre-employment chest $X$-ray should be carried out as a routine check before being contracted.

\section{Sample size}

169 participants were selected based on an LTBI prevalence of $12 \%$; confidence intervals were taken at $95 \%$ with a $5 \%$ margin of error. We added $10 \%$ more to the number in order to accommodate drop-outs or incomplete data. The total sample size consisted of 186 participants.

After oral consent was taken from the study population, all participants who agreed to participate in the study were included. Excluded from the study were pregnant women, nurses, the immunodeficient, administrative staff, technicians, contact with active TB patients within the previous three months and those with changes in their chest X-ray.

\section{Study tools}

Saudi guidelines do not recommend using IGRAs for the diagnosis of active tuberculosis and recommend TST for TB screening [8]. This study relies on the results of the TST reaction, and we considered $10 \geq \mathrm{mm}$ induration as positive in our study $[9,10]$.

A review of pre-employment records was done, including the results of TST and socio-demographic information, as well as potential factors influencing TST reaction (age, gender, marital status, medications, medical condition, smoking, BMI, BCG vaccination status, history of travel, contact with TB patients and review of chest $\mathrm{X}$-ray).

\section{Data analysis}

Data was analyzed using the IBM advanced SPSS statistical package version 20. Qualitative data was presented as frequencies and percentages, and the chi-square test (Fisher's exact test) was used to examine the relation between categorical variables, while continuous variables were presented as means and standard deviations, and the $t$-Test was used for comparison. Binary regression was used to analyze the risk factors. The statistical significance was determined using the $95 \% \mathrm{Cl}$ and $p<0.05$.

\section{Ethical consideration}

This study was approved by the Ethics and Research Review Committee of Umm Al-Qura Faculty of Medicine, and confidentiality was ensured during the study. The participants were approached and made clear of the nature and purpose of the study.

\section{Results}

Out of 186 personnel enrolled in this study, 70 (37.6\%) were newly hired medical doctors, 67 (36\%) medical interns, and $49(36.3 \%)$ were medical students. Approximately half of the study population was female $(99(53.2 \%))$. Most of the study subjects were single $(121(65.1 \%))$, with a minority being married $(63(33.9 \%))$. Most of the study population received BCG vaccination (164 (88.2\%)), while a minority of our study population were non-smokers, had no chronic diseases and were not on continuous medication use (25 (13.4\%), 28 (15.1\%), and 14 (7.5\%), respectively). Out of 186 participants tested in our study, 43 (23.1\%) were positive for PPD test reaction ( $\geq 10 \mathrm{~mm}$ induration) (Table 1).

\begin{tabular}{|l|l|l|}
\hline \multicolumn{3}{|l|}{ Table 1. Baseline characteristics of the 186 participants } \\
\hline & & $n(\%)$ \\
\hline Job & medical doctor & $70(37.6 \%)$ \\
& medical intern & $67(36 \%)$ \\
& medical student & $49(36.3 \%)$ \\
\hline Gender & male & $99(53.2 \%)$ \\
& female & $87(46.8 \%)$ \\
\hline Marital status & single & $121(65.1 \%)$ \\
& married & $63(33.9 \%)$ \\
& divorced or widowed & $2(1.1)$ \\
\hline Chronic disease & no & $158(84.9 \%)$ \\
& yes & $28(15.1 \%)$ \\
\hline Continuous medication use & no & $172(92.5 \%)$ \\
& yes & $14(7.5 \%)$ \\
\hline Smoking & no & $161(86.6 \%)$ \\
& yes & $25(13.4 \%)$ \\
\hline Received BCG vaccination & yes & $164(88.2 \%)$ \\
& no & $22(11.8 \%)$ \\
\hline Tuberculin skin test & negative & $143(76.9 \%)$ \\
& positive & $43(23.1 \%)$ \\
\hline
\end{tabular}

Additionally, the prevalence rate of LTBI showed no significant differences across job, gender, marital status, chronic disease, continuous medication use, smoking status, whether having received BCG vaccination, or with respect to age, with $p$-values of $0.543,0.969,0.712,0.091,0.876,0.534,0.963$ and 0.376 , respectively. On the other hand, the increased prevalence of LTBI in our study population was associated with increased BMI ( $p=0.046)$ (Table 2).

\begin{tabular}{|c|c|c|c|c|}
\hline & & \multicolumn{2}{|c|}{ Tuberculin skin test } & \multirow[t]{2}{*}{$p$} \\
\hline & & Negative & Positive & \\
\hline \multirow[t]{3}{*}{ Job } & medical doctor & $56(80.0 \%)$ & $14(20.0 \%)$ & \multirow[t]{3}{*}{0.543} \\
\hline & medical intern & $52(77.6 \%)$ & $15(22.4 \%)$ & \\
\hline & medical student & 35 (71.4\%) & $14(28.6 \%)$ & \\
\hline \multirow[t]{2}{*}{ Gender } & male & $67(77.0 \%)$ & $20(23.0 \%)$ & \multirow[t]{2}{*}{0.969} \\
\hline & female & $76(76.8 \%)$ & $23(23.2 \%)$ & \\
\hline \multirow[t]{3}{*}{ Marital status } & single & $92(76.0 \%)$ & $29(24.0 \%)$ & \multirow[t]{3}{*}{0.712} \\
\hline & married & $49(77.8 \%)$ & $14(22.2 \%)$ & \\
\hline & divorced or widow & $2(100.0 \%)$ & $0(0.0 \%)$ & \\
\hline
\end{tabular}




\begin{tabular}{|c|c|c|c|c|}
\hline & & \multicolumn{2}{|c|}{ Tuberculin skin test } & \multirow[t]{2}{*}{$p$} \\
\hline & & Negative & Positive & \\
\hline \multirow[t]{2}{*}{ Chronic disease } & no & $118(74.7 \%)$ & $40(25.3 \%)$ & \multirow[t]{2}{*}{0.091} \\
\hline & yes & $25(89.3 \%)$ & $3(10.7 \%)$ & \\
\hline \multirow[t]{2}{*}{ Continuous medication use } & no & $132(76.7 \%)$ & $40(23.3 \%)$ & \multirow[t]{2}{*}{0.876} \\
\hline & yes & $11(78.6 \%)$ & $3(21.4 \%)$ & \\
\hline \multirow[t]{2}{*}{ Smoking } & no & $125(77.6 \%)$ & $36(22.4 \%)$ & \multirow[t]{2}{*}{0.534} \\
\hline & yes & $18(72.0 \%)$ & $7(28.0 \%)$ & \\
\hline \multirow[t]{2}{*}{ Received BCG vaccination } & yes & $126(76.8 \%)$ & $38(23.2 \%)$ & \multirow[t]{2}{*}{0.963} \\
\hline & no & $17(77.3 \%)$ & $5(22.7 \%)$ & \\
\hline Age & & $\begin{array}{l}\text { mean } \pm S D \\
27.34 \pm 7.04\end{array}$ & $\begin{array}{l}\text { mean } \pm S D \\
26.25 \pm 7.02\end{array}$ & 0.376 \\
\hline BMI & & $\begin{array}{l}\text { mean } \pm S D \\
25.24 \pm 6.76\end{array}$ & $\begin{array}{l}\text { mean } \pm S D \\
27.46 \pm 4.77\end{array}$ & 0.046 \\
\hline
\end{tabular}

\begin{tabular}{|l|l|l|l|l|l|}
\hline \multirow{2}{*}{ Table 3. Binary regression analysis of risk factors for latent tuberculosis infection in healthcare workers } \\
\cline { 2 - 6 } & B & Odds ratio & $p$ & \multicolumn{3}{l|}{ 95\% CI for EXP (B) } \\
\hline Age & -0.028 & 0.973 & & Lower & Upper \\
\hline Gender - male & 0.178 & 1.195 & 0.371 & 0.915 & 1.034 \\
\hline Marital status - married & -0.115 & 0.891 & 0.668 & 0.530 & 2.694 \\
\hline Chronic disease & 1.574 & 4.828 & 0.812 & 0.345 & 2.303 \\
\hline Medications & -1.125 & 0.325 & 0.064 & 0.910 & 25.608 \\
\hline Smoker & -0.315 & 0.730 & 0.243 & 0.049 & 2.147 \\
\hline BMI & 0.068 & 1.071 & 0.552 & 0.259 & 2.060 \\
\hline BCG vaccination & -0.242 & 0.785 & 0.039 & 1.003 & 1.142 \\
\hline
\end{tabular}

Binary regression analyses showed that TST+ was significantly associated with BMI $(\mathrm{OR}=1.071,95 \% \mathrm{Cl}$ : $1.003-1.142$, $p=0.039$ ), while it was not significantly associated with male gender (OR $=1.195,95 \% \mathrm{Cl}: 0.530-2.694, p=0.668)$, age (OR $=0.973,95 \% \mathrm{Cl}: 0.915-1.034, p=0.371)$, smoking $(\mathrm{OR}=0.730$, 95\% Cl: $0.259-2.060, p=0.552)$, BCG vaccination (OR $=0.785$, $95 \% \mathrm{Cl}: 0.249-2.481, p=0.680)$ or chronic disease $(\mathrm{OR}=4.828$, 95\% Cl: 0.910-25.608, $p=0.064$ ) (Table 3).

\section{Discussion}

Respiratory infections and other infectious diseases, due to close contacts, are a challenging hazard to HCWs. Tuberculosis is still considered a worldwide health problem. It is one of the most important and leading causes of morbidity and mortality in several areas of the world. The last population-based survey studying the epidemiology of tuberculosis infection in Saudi Arabia was published by Balkhy et al. in 2017. Worldwide research, including in Saudi Arabia, shows an increased risk of tuberculosis as a re-emerging disease [11].

The WHO reported the prevalence of TB in Saudi Arabia to be 86 per 100,000, 55 per 100,000, 10.6 per 100,000 and 10 per 100,000 in 1990, 2004, 2015 and 2017, respectively, compared to the worldwide average of 133 per 100,000 [11, 12].

The current study reviewed the results of PPD testing in 186 persons, including newly hired doctors, medical interns and medical students, with a positive PPD finding in 43 (23.1\%) of the participants.

Local studies in Saudi Arabia showed a wide range of LTBI prevalence [11]. In a tertiary hospital in Riyadh, Hajoj et al. screened all newly hired HCWs from 2012 to 2015 and found that $31.5 \%$ of the included HCWs were positive [13]. This higher rate could be due to the TB endemic region of the enrolled $\mathrm{HCWs}$. El-Helaly et al. reviewed the health records of new HCWs from 2009 to 2011 , which revealed that $29.8 \%$ had a positive PPD [14]. A recent study in 2020 found 6,090 out of $6,404 \mathrm{HCWs}$ (95.1\%) tested utilizing TST had a negative PPD, while 314 (4.9\%) had a positive PPD [15]. Another study in Saudi Arabia using both TST and QFT GIT among healthcare workers working during the Hajj pilgrimage season found that the LTBI rate was $10.8 \%$ [16]. From Muhayil city, another study was carried out in the same context using TST testing $208 \mathrm{HCWs}$ and found that 47 (22.5\%) tested positive [17]. Murad et al. found that out of 296 students, 35 undergraduate female students of health sciences (12\%) had a positive PPD reaction [18].

A study in two Moroccan cities reported the prevalence of LTBI among $631 \mathrm{HCWs}$ to be varied between $40.7 \%$ with QFT-GIT to $52 \%$ based on TST [19]. Additionally, Al-Sokkary et al. conducted a study on HCWs in a chest hospital in Zagazig city, Egypt, finding that the prevalence of LTBI identified by QFT-GIT and by TST was $28.8 \%$ and $59.1 \%$, respectively [20].

In the current study, we observed that out of 67 medical intern, 15 (22.4\%) had a positive TST, which is nearly similar to a report from a study carried out in Tunisia that found that the prevalence of LTBI was $26.6 \%$ among medical interns [21].

Cohen et al., in 2019, systematically reviewed 88 studies from 36 countries and found that with a $10 \mathrm{~mm}$ cut-off, the total prevalence of LTBI was $24.8 \%$ based on IGRA, whereas it was $21.2 \%$ based on TST. They concluded that with significant regional differences, the international prevalence of LTBI no longer amounts to a third of the world population but is closer to one-fourth [22]. Another systematic review done by Alele et al. screened 21 studies and found that the median prevalence of LTBI among HCWs was 62\% [23].

LTBI prevalence in our study is less than reported by Chen et al., who concluded that a $33.9 \%$ of HCWs in chest hospitals had positive PPD; these higher results may be due to increased exposure of HCWs to TB bacilli inside the chest hospitals [24]. Furthermore, a work from India by Janagond et al. reported 
higher positive PPD results (36.8\%) [25]; this higher rate may be due to India being ranked by WHO as one of the top eight countries having a high incidence of TB [26]. These values are compatible with the prevalence reported from high TB burden countries such as Brazil (37\%), China (54\%) and India (43\%) [27].

On the other hand, the study by Li et al. reviewed the UK occupational TB HCWs screening database from 2010 to 2017 and reported a $4 \%$ positive PPD rate among new HCWs. This lower rate than our study finding may be explained by UK being one of the low incidence TB countries [28]. Similarly, the results of a retrospective study in Italy by Olivieri et al., which screened 2,136 HCWs, found 144 (6.7\%) were PPD positive, which is less than our findings [29].

Uden et al. reported the prevalence of LTBI among healthcare workers [HCWs] of $37 \%$ [30]. Additionally, Parks, in South Korea, documented that the prevalence of LTBI was higher among HCWs compared to the general population [31]. Keshavarz et al., in 2019, reported that the LTBI prevalence among HCWs in the Children's Care Hospital in Iran to be $27.13 \%$ based on TST [32]. Apriani et al., in 2019, searched multiple databases, such as Embase, Medline and Web of Science, for publications on LTBI among HCWs from 2015 to 2017 and concluded that prevalence of a positive TST was 14-98\% [33]. A study from Côte D'Ivoire in Africa by Kassim et al. reported a very high LTBI prevalence; out of the 512 enrolled HCWs, 405 (79\%) had a positive PPD reaction [34].

The current study demonstrated that the prevalence rate of LTBI showed no significant differences across job, gender, marital status, chronic disease, continuous medication use, smoking, whether having received BCG vaccination and with respect to age, with $p$-values of $0.543,0.969,0.712,0.091,0.876,0.534$, 0.963 and 0.376 , respectively. On the other hand, the increased prevalence of $L T B I$ in our study population was associated with increased BMI $(p=0.046)$.

Out of 49 undergraduate medical students enrolled in the current study, 14 (28.6\%) had a positive TST, which was much higher than an Italian study showing that among 2,082 undergraduates, $23(1.1 \%)$ had a positive TST [35]. Additionally 14 (20\%) of our medical doctors and $22.4 \%$ of our medical interns had a positive TST, which is much higher than the same Italian study, which revealed that among 864 postgraduates, 24 $(2.78 \%)$ had a positive TST. LTBI was statistically significant when comparing postgraduates to undergraduates in the Italian study, while our study showed no significance. The Italian study found that the number of previously vaccinated individuals was more than non-vaccinated ( $p=0.001)$ in the postgraduate group, while our study found no significant difference.

In our study, we found that LTBI was $23 \%$ among males and $23.2 \%$ among females, with no statistically significant difference. A similar result was reported by Nabil et al., with $22 \%$ in males and $23 \%$ in females [36]. Schablon et al. reported no significant difference $-7.2 \%$ in males and $7.1 \%$ in females [37]. Additionally, the previously mentioned Italian study found that there was no significant statistical gender difference regarding the incidence of LTBI [35]. On the other hand, further studies showed that the male gender is at risk for active TB infection due to more exposure, as well as the biological differences between the sexes [38, 39]. Some studies documented Asian and African ethnicities, female gender and younger age groups as associated TB risk factors $[40,41]$. Similarly, a study by Mirtskhulava et al. reported that women had a higher prevalence of LTBI than men [42].

Our study showed no difference between smokers and non-smokers having LTBI; however, other studies reported that smokers had a higher risk of LTBI when assessed by TST $[43,44]$.

This study found no association between age and LTBI, while other studies reported an increased risk of LTBI prevalence at an elderly age $[45,46]$. Nienhaus et al., in 2007, documented that the prevalence rate of LTBI increased from $4.6 \%$ in participants younger than 30 years to $69.2 \%$ in those older than 60 years [47].

Marital status in our study was not a risk factor for LTBI, but other studies showed that divorced/widowed individuals had the highest prevalence, while single individuals had the lowest prevalence (30.3\% vs $4.2 \%)$ [11].

Body mass index (BMI) has been shown to be linked to an individual's susceptibility to numerous infections and conditions. However, the association between BMI and the risk of TB infection has been poorly studied; our current study demonstrated that the increased prevalence of LTBI is associated with increased BMI $(p=0.046)$. The results of a systematic review done by Saag et al. found that underweight individuals are not at increased risk of LTBI [48]. Another study from a rural area in China revealed that $\mathrm{BMI} \geq 28.0 \mathrm{~kg} / \mathrm{m}^{2}$ was found to be independently associated with an individual's susceptibility to TB infection [49].

The link between BMI and TB infection is not widely understood, despite years of research on its association with active TB $[50,51]$. It is thought that increased adipose tissue negatively affects immune function and decrease host defense in obese persons [52]. Additionally, the accumulated adiposity could decrease the pulmonary defense via metabolic disturbances [53].

\section{Conclusions}

The prevalence of LTBI in our study was considered high. For this reason, strong infection control precautions are strongly recommended to prevent TB infection. Furthermore, more studies are needed to assess the adherence and practices of healthcare workers, especially those who are exposed to TB patients. There was a positive relationship between obesity and LTBI. Accordingly, more studies are recommended to study the relation between LTBI and active TB, and we suggest adding obesity to the high risk group for TB. Family physicians and primary healthcare providers play an important role in early diagnosis of LTBI and in health education.

Source of funding: This work was funded from the authors' own resources.

Conflicts of interest: The authors declare no conflicts of interest.

\section{References}

1. Houben RMGJ, Dodd PJ. The Global Burden of Latent Tuberculosis Infection: a re-estimation using mathematical modelling. PLoS MED 2016; 13(10): e1002152.

2. Vynnycky E, Fine PE. The natural history of tuberculosis: the implications of age-dependent risks of disease and the role of reinfection. Epidemiol Infect 1997; 119(2): 183-201.

3. WHO. Global tuberculosis report 2018 [cited 28.02.2019]. Available from URL: http://www.who.int/tb/publications/global_report/en/.

4. Al-Hajoj S, Varghese B. Tuberculosis in Saudi Arabia: the journey across time. J Infect Dev Ctries 2015; 9(3): 222-231.

5. Menzies D, Joshi R, Pai M. Risk of tuberculosis infection and disease associated with work in health care settings. Int J Tuberc Lung Dis 2007; 11(6): 593-605.

6. American Thoracic Society. Targeted tuberculin testing and treatment of latent tuberculosis infection. MMWR Recomm Rep 2000; 49(6): 1-51. 
7. Getahun H, Matteelli A, Chaisson RE, et al. Latent Mycobacterium tuberculosis infection. N Engl J Med 2015; 372(22): $2127-2135$.

8. Al Jahdali HH, Baharoon S, Abba AA, et al. Saudi guidelines for testing and treatment of latent tuberculosis infection. Ann Saudi Med 2010; 30(1): 38-49.

9. American Thoracic Society, Centers for Disease Control and Prevention. Targeted tuberculin testing and treatment of latent tuberculosis infection. Am J Respir Crit Care Med 2000; 49(6): 1-51.

10. Joshi R, Reingold AL, Menzies D, et al. Tuberculosis among health-care workers in low - and middle income countries: a systematic review. PLOS MED 2006; 3: e494.

11. Balkhy HH, ElBeltagy K, El-Saed A, et al. Prevalence of Latent Mycobacterium Tuberculosis infection (LTBI) in Saudi Arabia. Population based survey. Int J Infect Dis 2017; 60: 11-16.

12. World Health Organization (WHO). Global tuberculosis report 2017. Geneva: WHO, 30 Oct 2017. Available from URL: http://www.who. int/mediacentre/news/releases/2017/political-commitment-tb/en/.

13. Al Hajoj S, Varghese B, Datijan A, et al. Interferon gamma release assay versus tuberculin skin testing among healthcare workers of highly diverse origin in a moderate tuberculosis burden country. PLOS ONE 2016; 11(5): e0154803.

14. El-Helaly M, Khan W, El-Saed A, et al. Pre-employment screening of latent tuberculosis infection among healthcare workers using tuberculin skin test and QuantiFERON-TB Gold test at a tertiary care hospital in Saudi Arabia. J Infect Public Health 2014; 7(6): 481-488.

15. Kofi M, Rsheed A, AlBattal A, et al. Screening for latent TB among HCWs newly hired in a major hospital in Riyadh, Saudi Arabia. J Infect Public Health 2020; 6(3): 10-15.

16. Bukhary ZA, Amer SM, Emara MM, et al. Screening of latent tuberculosis infection among health care workers working in Hajj pilgrimage area in Saudi Arabia, using interferon gamma release assay and tuberculin skin test. Ann Saudi Med 2018; 38(2): 90-96.

17. Nabil H, Hosam-Eldin H. Prevalence of latent tuberculosis infection among multinational healthcare workers in Muhayil Saudi Arabia. Egypt J Broncho 2015; 9: 183-187.

18. Murad M, Abdulmageed S. Tuberculosis screening among health sciences students in Saudi Arabia in 2010. Ann Saudi Med 2012; 32(5): 527-529.

19. Sabri A, Quistrebert J, Naji H. et al. Prevalence and risk factors for latent tuberculosis infection among healthcare workers in Morocco. PLOS ONE 2019; 14(8): e0221081.

20. El-Sokkary R, Abu-Taleb A, El-Seifi O, et al. Assessing the prevalence of latent tuberculosis among health care providers in Zagazig City, Egypt using tuberculin skin test and Quantiferon-TB Gold In-Tube Test. Cent Eur J Public Health 2015; 23(4): 324-330.

21. Toujani S, Cherif J, Mjid M, et al., Evaluation of tuberculin skin test positivity and early tuberculin conversion among medical intern trainees in Tunisia. Tanaffos 2017; 16: 149-156.

22. Cohen A, Mathiasen VD, Schön T, et al. The global prevalence of latent tuberculosis: a systematic review and meta-analysis. Eur Respir J 2019; 54(3): 1900655.

23. Alele F, Franklin R, Emeto $\mathrm{T}$, et al. Occupational tuberculosis in healthcare workers in sub-Saharan Africa: a systematic review. Arch Environ Occup Health 2019; 74(3): 95-108.

24. Chen B, Gu H, Wang X, et al. Prevalence and determinants of latent tuberculosis infection among frontline tuberculosis healthcare workers in southeastern China: a multilevel analysis by individuals and health facilities. Int J Infect Dis 2018; 79: 26-33.

25. Janagond A, Ganesan V, Vijay G, et al. Screening of health-care workers for latent tuberculosis infection in a Tertiary Care Hospital. Int J Mycobacteriol 2017; 6(3): 253-257.

26. World Health Organization. Global Tuberculosis Report. Geneva: WHO; 2019.

27. Nasreen S, Shokoohi M, Malvankar-Mehta M. Prevalence of latent tuberculosis among health care workers in high burden countries: a systematic review and meta-analysis. PLOS ONE 2016; 11: e0164034.

28. Li M, Schwec M, Clerk N, et al. Occupational tuberculosis screening for healthcare workers in a UK center. Eur Respir J 2018; 52 : PA3876.

29. Olivieri R, Scarnera S, Ciabattini A, et al. Using IFN-gamma release assay to confirm tuberculin skin test improves the screening of latent tuberculosis infection in Italian healthcare workers. J Occup Med Toxicol 2016; 11: 29.

30. Uden L, Barber E, Ford N, et al. Risk of tuberculosis infection and disease for health care workers: an updated meta-analysis. Open Forum Infect Dis 2017; 4(3): ofx137.

31. Park JS. The prevalence and risk factors of latent tuberculosis infection among health care workers working in a Tertiary Hospital in South Korea. Tuberc Respir Dis (Seoul) 2018; 81(4): 274-280.

32. Keshavarz S, Mahmoudi S, Pourakbari B, et al. Screening of healthcare workers for latent tuberculosis infection in the low tuberculosis burden country: QuantiFERON-TB gold in tube test or tuberculin skin test? Arch Environ Occup Health 2019; 74(3): $109-114$.

33. Apriani L, McAllister S, Sharples K, et al. Latent tuberculosis infection in healthcare workers in low- and middle-income countries: an updated systematic review. Eur Respir J 2019; 53(4): 1801789.

34. Kassim S, Zuber P, Wiktor SZ, et al. Tuberculin skin testing to assess the occupational risk of Mycobacterium tuberculosis infection among health care workers in Abidjan, Côte d'Ivoire. Int J Tuberc Lung Dis 2000; 4: 321-326.

35. Verso M, Serra N, Ciccarello A, et al. Latent tuberculosis infection among healthcare students and postgraduates in a Mediterranean Italian area: what correlation with work exposure? Int J Environ Res Public Health 2019; 17(1): 137.

36. Nabil A. Abdelghaffar $\mathrm{H}$, Hosam-Eldin $\mathrm{H}$. Prevalence of latent tuberculosis infection among multinational healthcare workers in Muhayil Saudi Arabia. Egypt J Broncho 2015; 9: 183-187.

37. Schablon A, Beckmann G, Harling M, et al. Prevalence of latent tuberculosis infection among health care workers in a hospital for pulmonary diseases. J Occup Med Toxicol 2009; 4: 1.

38. Horton K, MacPherson P, Houben RMGJ, et al. Sex differences in tuberculosis burden and notifications in low- and middle-income countries: a systematic review and meta-analysis. PLOS MED 2016; 13: e1002119.

39. Neyrolles O, Quintana-Murci L. Sexual inequality in tuberculosis. PLoS MED 2009; 6(12): e1000199.

40. García-Rodríguez J, Álvarez-Díaz H, Lorenzo-García M, et al. Extrapulmonary tuberculosis: epidemiology and risk factors. Enferm Infecc Microbiol Clin 2011; 29(7): 502-509.

41. Al-Otaibi F, El Hazmi MM. Extra-pulmonary tuberculosis in Saudi Arabia. Indian J Pathol Microbiol 2010; 53(2): $227-231$.

42. Mirtskhulava V, Kempker R, Shields $\mathrm{K}$, et al. Prevalence and risk factors for latent tuberculosis infection among health care workers in Georgia. Int J Tuberc Lung Dis 2008; 12: 513-519.

43. De Souza FM, do Prado TN, Pinheiro Jdos S, et al. Comparison of interferon- $\gamma$ release assay to two cut-off points of tuberculin skin test to detect latent Mycobacterium tuberculosis infection in primary health care workers. PLOS ONE 2014; 9(8): e102773.

44. He GX, Wang LX, Chai SJ, et al. Risk factors associated with tuberculosis infection among health care workers in Inner Mongolia, China. Int J Tuberc Lung Dis 2012; 16: 1485-1491.

45. Whitaker JA, Mirtskhulava V, Kipiani M, et al. Prevalence and incidence of latent tuberculosis infection in georgian healthcare workers. PLOS ONE 2013; 8: e58202. 
46. Babayigit C, Ozer B, Ozer C, et al. Performance of QuantiFERON-TB Gold In-Tube test and Tuberculin Skin Test for diagnosis of latent tuberculosis infection in BCG vaccinated health care workers. Med Sci Monit 2014; 20: 521-529.

47. Nienhaus A, Schablon A, Loddenkemper R, et al. Prevalence of latent tuberculosis infection in healthcare workers in geriatric care. Pneumologie 2007; 61: 613-616.

48. Saag LA, LaValley MP, Hochberg NS, et al. Low body mass index and latent tuberculous infection: a systematic review and meta-analysis. Int J Tuberc Lung Dis 2018; 22(4): 358-365.

49. Zhang $\mathrm{H}$, Li X, Xin H, et al. Association of Body Mass Index with the tuberculosis infection: a population-based study among 17796 adults in rural China. Sci Rep 2017; 7: 41933.

50. Hanrahan CF, Golub JE, Mohapi L, et al. Body mass index and risk of tuberculosis and death. AIDS 2010; 24(10): 1501-1508.

51. Maro I, Lahey T, MacKenzie T, et al. Low BMI and falling BMI predict HIV-associated tuberculosis: a prospective study in Tanzania. Int J Tuberc Lung Dis 2010; 14(11): 1447-1453.

52. Milner JJ, Beck MA. The impact of obesity on the immune response to infection. Proc Nutr Soc 2012; 71(2): 298-306.

53. Mancuso P. Obesity and respiratory infections: does excess adiposity weigh down host defense? Pulm Pharmacol Ther 2013; 26(4): 412-419.

Tables: 3

Figures: 0

References: 53

Received: 18.07.2020

Reviewed: 31.07 .2020

Accepted: 8.03.2021

Address for correspondence:

Reda Goweda, MD, PhD, Assoc. Prof.

Department of Family Medicine

Faculty of Medicine

Suez Canal University

Postal code 41522

Ismailia

Egypt

Mobile: +201003583933

E-mail: redagoweda@yahoo.com 